\title{
MODEL CONSIDERING RESIDUAL STIFFNESS AND STIFFNESS DISCONTINUITY OF BOLTED JOINTS
}

\author{
Sheng-Ao Wang, Min Zhu, Zi-Jian Xu, Ming Guo, Biao Li, Fei Wu \\ College of Nuclear Science and Technology, Naval University of Engineering, Wuhan, China \\ e-mail: min0zhu@163.com (corresponding author)
}

\begin{abstract}
The nonlinear dynamic behavior has an important impact on energy dissipation and vibration damping characteristics of bolted joints. Firstly, the development of tangential dynamic models is summarized and analyzed. Secondly, a five-parameter Iwan model based on a truncated power-law distribution is proposed. The backbone and hysteresis curves are obtained. Thirdly, normalized and dimensionless analysis is performed. On the basis of the above, a more concise four-parameter Iwan model with stiffness continuity is proposed. Finally, the validity of the model is verified by comparing the energy dissipation vs excitation force amplitude curve with the experimental data.
\end{abstract}

Keywords: nonlinear dynamic behaviour, Iwan model, energy dissipation

\section{Introduction}

Mechanical joints have a significant effect on structural dynamics. Bolted joints cause local stiffness and damping changes and are often the primary source of energy dissipation and damping in assembled structures. In many such structures, the damping due to relative interfacial joint motion accounts for as much as $90 \%$ of the total energy (Beards, 1992). The bolted joint has been widely used in aerospace, weaponry, automobile manufacturing and other industrial fields with the advantages of simple structures, with convenient assembly and disassembly, etc. However, it causes complex friction and slip behaviour as shown in Fig. 1. Insufficient understanding and failed design of bolted joints have resulted in serious aircraft engine failures and batch recalls of automobiles. Bolt engineering has become one the most important and unresolved general safety issues (Bickford, 2007).

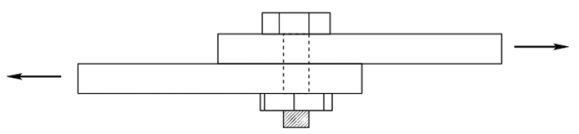

Fig. 1. Tangential excitation on bolted joints

Generally, numerical simulation can be used to analyse the influence of bolted joints on dynamic characteristics of the overall structure, but it is only feasible for simple structure analysis. When it comes to complex assemblies, the detailed bolt finite element model requires certain unit element size of the overall structure, which brings great difficulties to the calculation. Therefore, it is also important to study the nonlinear dynamic behaviour of bolted joints and reduce the calculation scale of simulation (Segalman et al., 2009).

There are two types of approaches in this field. The first category includes fractal characterization and statistical summation methods based on geometric and physical parameter modelling. The other category includes phenomenological models such as the Iwan model (Iwan, 1966) and the Valanis model (Ferri, 1995) based on model assumptions and parameter identification (Cao 
et al., 2020). Although the research based on the physical mechanism can describe the nonlinear dynamic behaviour from the mechanism, the phenomenological method of the simple model and easy-to-identify parameters have higher engineering application. Due to systematic description of the slip processing the Iwan model, it has been widely concerned and developed by many scholars (Argatov and Butcher, 2011). Segalman proposed a four-parameter Iwan model based on a truncated power-law spectrum, by summarizing the observation that the power of energy dissipation and loading force amplitude is between 2.0 and 3.0 (Goodman, 1959; Mindlin, 1949, 1952). Song et al. (2004) paralleled the Iwan model with a linear spring to characterize the residual stiffness after macroslip, and applied it to the AIBE (adjusted Iwan beam element). Zhang et al. (2012) derived a normalized calculation formula for periodic energy dissipation of the classic Iwan model, and a justified Iwan model proposed was by Song. Li et al. (2015) introduced a six-parameter Iwan model based on a double-pulse non-uniform density truncated power law distribution, and verified its validity. $\mathrm{Li}$ and $\mathrm{Xu}$ (2017) studied the influence of a normal load on the mechanical behaviour between interfaces. Wang and Fan (2019) studied the influence of excitation amplitudes on the nonlinear dynamic behaviour, and derived an equation for the local nonlinear connection. Based on the discrete Iwan model, Liu et al. (2020) verified the agreement between the equivalent finite element model and the contact model.

\section{Comparative analysis of typical Iwan models}

The classic Iwan model is divided into a parallel-series model and a series-series model, among which the parallel-series model has better applicability. The parallel-series Iwan model has two assumptions. One is the parallel model of the Jenkins element composed of a spring and a friction resistor in series, as shown in Fig. 2a. The other is that the yield force obeys the band-limited distribution as shown in Fig. 2b, where $\varphi\left(f^{*}\right)$ represents the distribution function of the yield force $f^{*}, f_{y}$ is the center value of the band-limited distribution (the average yield force), and $\Delta f$ is the bandwidth.

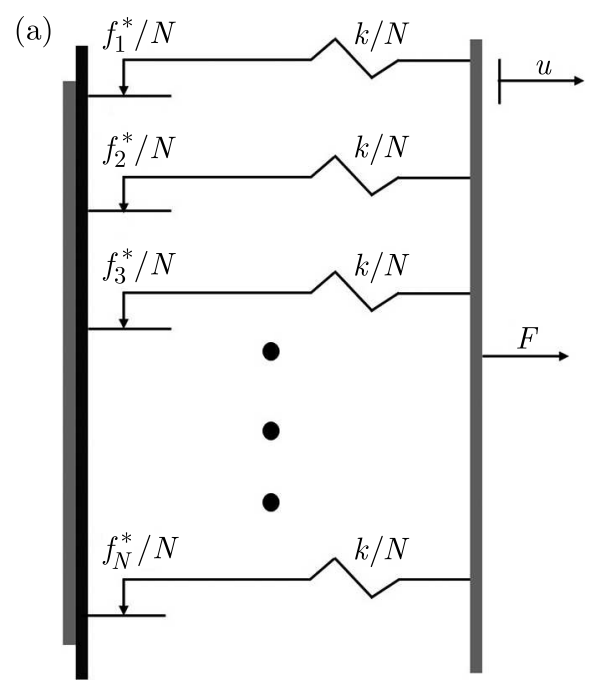

(b)

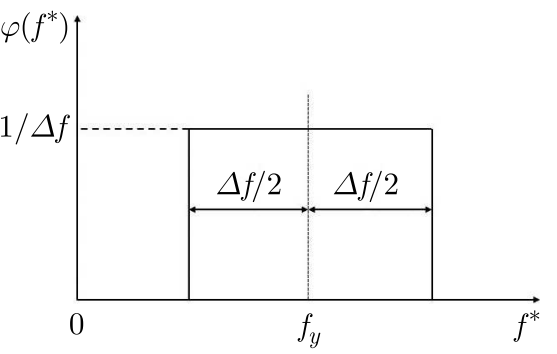

Fig. 2. Two assumptions of the classic Iwan model: (a) parallel-series model, (b) distribution function $\varphi\left(f^{*}\right)$

Mathematically, the discrete form of the backbone curve in the classic Iwan model is

$$
f=\sum_{i=1}^{n} \frac{f_{i}^{*}}{N}+\frac{k u(N-n)}{N}
$$


where $u$ is the imposed displacement in the initial loading, $k$ is the total stiffness of all Jenkins elements, $n$ is the number of Jenkins elements that yield under the condition of displacement $u$. The yield forces of Jenkins elements are arranged in order as $f_{1}^{*}<f_{2}^{*}<\cdots<f_{N}^{*}$.

When $N$ tends to infinity, Eq. (2.1) can be written in the equivalent form

$$
f=\int_{0}^{k u} f^{*} \varphi\left(f^{*}\right) d f^{*}+k u \int_{k u}^{\infty} \varphi\left(f^{*}\right) d f^{*}
$$

The restoring force of the unloading process of the hysteretic curve is

$$
\overleftarrow{f}(u)=\int_{0}^{\frac{k(A-u)}{2}}-f^{*} \varphi\left(f^{*}\right) d f^{*}+\int_{\frac{k(A-u)}{2}}^{k A}\left[k u-\left(k A-f^{*}\right)\right] \varphi\left(f^{*}\right) d f^{*}+k u \int_{k A}^{\infty} \varphi\left(f^{*}\right) d f
$$

Through the Masing criterion, the restoring force-displacement relationship during the loading process of the hysteretic curve can be obtained as

$$
\vec{f}(u)=-\overleftarrow{f}(-u)
$$

When a monotonic load is applied, the stiffness of the backbone curve degrades to zero after reaching the maximum yield strength of $f_{y}+\Delta f / 2$, which is inconsistent with Gaul's (1997) experimental results. Song added a spring with stiffness $k_{a}$ to characterize the residual stiffness after macroslip. The modified Iwan model is shown in Fig. 3, where $k_{r}=k-k_{a}$, and $k$ is the initial stiffness. Equations (2.1), (2.2) and (2.3) now become

$$
\begin{aligned}
& f_{\text {adjusted }}=\sum_{i=1}^{n} \frac{f_{i}^{*}}{N}+\frac{k_{r} u(N-n)}{N}+k_{a} u \\
& f_{\text {adjusted }}=\int_{0}^{k_{r} u} f^{*} \varphi\left(f^{*}\right) d f^{*}+k_{r} u \int_{k_{r} u}^{\infty} \varphi\left(f^{*}\right) d f^{*}+k_{a} u \\
& \overleftarrow{f}_{\text {adjusted }}(u)=\int_{0}^{\frac{k_{r}(A-u)}{2}}-f^{*} \varphi\left(f^{*}\right) d f^{*}+\int_{\frac{k_{r}(A-u)}{2}}^{k_{r} A}\left[k_{r} u-\left(k_{r} A-f^{*}\right)\right] \varphi\left(f^{*}\right) d f^{*} \\
& +k_{r} u \int_{k A}^{\infty} \varphi\left(f^{*}\right) d f+k_{a} u
\end{aligned}
$$

Goodman (1959) pointed out that the energy dissipation caused by oscillatory lateral loads imposed on two spheres pushed together yields a power-law slope of 3.0 in the regime of small lateral loads. Segalman of Sandia National Laboratory proposed truncated power-law spectra, as shown in Fig. 4.

Table 1 shows a comparison of the three models.

Scholars have also revised the model to address these problems. For example, Li et al. (2015) proposed a six-parameter model through the modified double-pulse truncated power-law distribution. However, this model has many parameters to be identified and does not directly consider the stiffness continuity. Therefore, this paper combines the linear spring to characterize the residual stiffness and the truncated power-law distribution to represent the power relationship between energy dissipation and the displacement amplitude. First, a five-parameter model considering residual stiffness is proposed. On this basis, the residual stiffness is further considered, and a concise adjusted four-parameter model is obtained. 


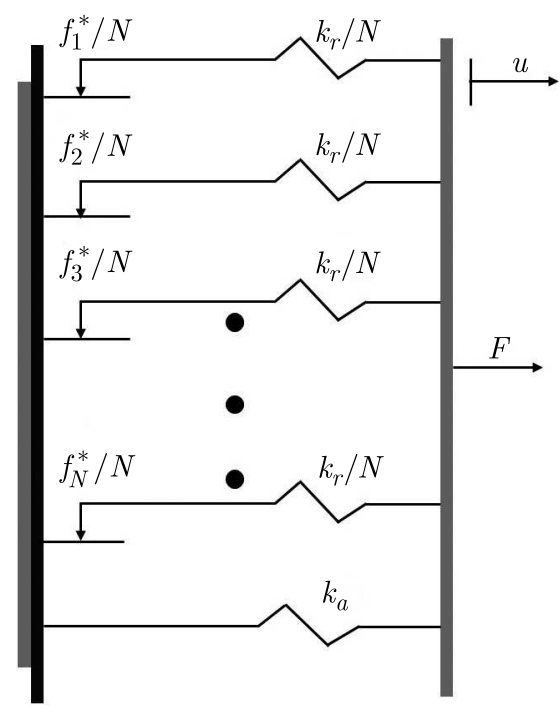

Fig. 3. Modified Iwan model

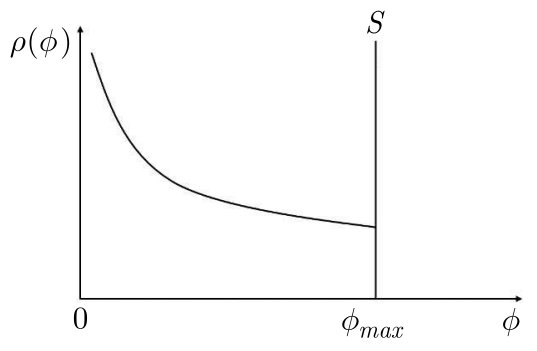

Fig. 4. Truncated power-law spectra

Table 1. Features and deficiencies of the three models

\begin{tabular}{|c|c|c|c|}
\hline Model type & Classic Iwan model & Justified Iwan model & $\begin{array}{c}\text { Four-parameter } \\
\text { Iwan model }\end{array}$ \\
\hline \hline Constitution & Jenkins elements & $\begin{array}{c}\text { Jenkins elements and } \\
\text { linear spring }\end{array}$ & Jenkins elements \\
\hline $\begin{array}{c}\text { Distribution } \\
\text { function }\end{array}$ & $\begin{array}{c}\text { Band-limited } \\
\text { distribution }\end{array}$ & $\begin{array}{c}\text { Band-limited } \\
\text { distribution }\end{array}$ & $\begin{array}{c}\text { Truncated power-law } \\
\text { distribution }\end{array}$ \\
\hline Deficiencies & $\begin{array}{c}\text { No residual stiffness, } \\
\text { stiffness continuity and } \\
\text { power law relation }\end{array}$ & $\begin{array}{c}\text { No stiffness continuity } \\
\text { and power } \\
\text { law relation }\end{array}$ & $\begin{array}{c}\text { No residual stiffness } \\
\text { and stiffness } \\
\text { continuity }\end{array}$ \\
\hline
\end{tabular}

\section{Five-parameter model considering residual stiffness}

In order to characterize the residual stiffness, this paper proposes to use a linear spring with stiffness $k_{a}$ to characterize the residual stiffness and to modify the Jenkins element stiffness to $k_{r}=K_{T}-k_{a}$. Through the combination of spring stiffness modification and truncated power-law distribution, the goodness of the Segalman model can be retained in microslip, and the disablility for residual stiffness can be eliminated. The core assumptions of the five-parameter model are shown in Fig. 5, where $K_{T}$ is the initial stiffness, $\phi=f^{*} / k_{r}$ is the yield displacement of Jenkins elements, and $\rho(\phi)=k^{2} \varphi\left(f^{*}\right)$ is the distribution function of $\phi$. 

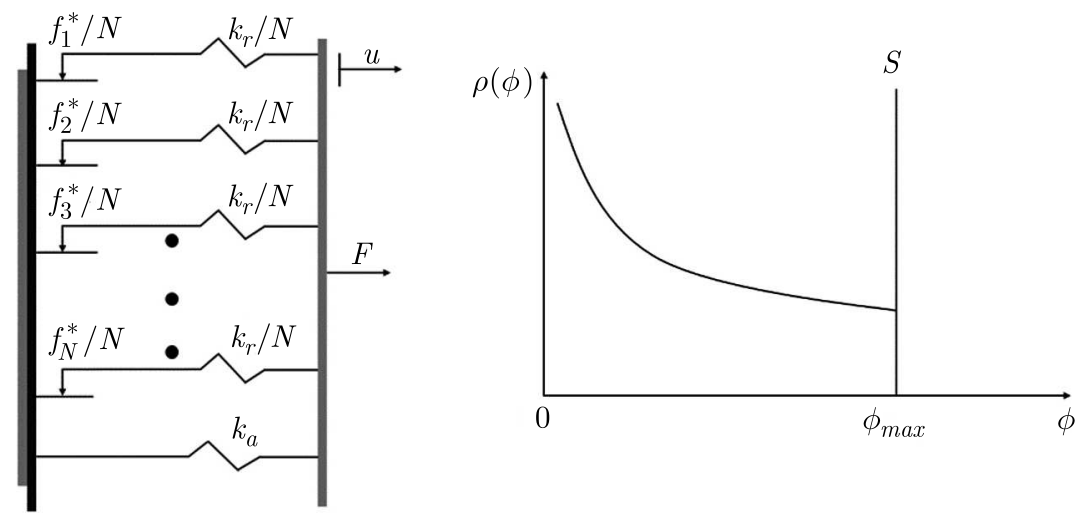

Fig. 5. Five-parameter model

Referring to Eq. (2.5) 2 , the restoring force becomes

$$
F(u)=\int_{0}^{u} \phi \rho(\phi) d \phi+\int_{u}^{\infty} u \rho(\phi) d \phi+k_{a} u
$$

The density function of the truncated power-law distribution is

$$
\rho(\phi)=k^{2} \varphi\left(f^{*}\right)=R \phi^{\chi}\left[H(\phi)-H\left(\phi-\phi_{\max }\right)\right]+S \delta\left(\phi-\phi_{\max }\right)
$$

Substituting Eq. (3.2) into Eq. (3.1), we can get

$$
F(u)= \begin{cases}\left(\frac{R \phi_{\max }^{\chi+1}}{\chi+1}+S+k_{a}\right) u-\frac{R u^{\chi+2}}{(\chi+1)(\chi+2)} & u<\phi_{\max } \\ k_{a} u+S \phi_{\max }+R \frac{\phi_{\max }^{\chi+2}}{\chi+2} & u \geqslant \phi_{\max }\end{cases}
$$

Deriving Eq. (3.3) with respect to $u$, the outcome is

$$
K(u)= \begin{cases}\frac{R\left(\phi_{\max }^{\chi+1}-u^{\chi+1}\right)}{\chi+1}+S+k_{a} & u<\phi_{\max } \\ k_{a} & u \geqslant \phi_{\max }\end{cases}
$$

It can be seen from Eq. (2.4) that the initial stiffness can be expressed as

$$
K_{T}=\frac{R \phi_{\max }^{\chi+1}}{\chi+1}+S+k_{a}
$$

The macroslip force is

$$
F_{s}=\int_{0}^{\infty} \phi \rho(\phi) d \phi+k_{a} \phi_{\max }=\phi_{\max }\left(\frac{R \phi_{\max }^{\chi+1}}{\chi+1}\right)\left(\frac{\chi+1}{\chi+2}+\beta\right)
$$

where $\beta=\left(S+k_{a}\right) /\left[R \phi_{\max }^{\chi+1} /(\chi+1)\right]$. This model can be determined by five parameters: $F_{s}, K_{T}$, $k_{a}, \chi$ and $\beta$. The first three parameters have clear physical meanings and can be measured. The rest two parameters can be identified by fitting with experimental data.

The parameters $R, \phi_{\max }, S$ can be expressed as

$$
\begin{aligned}
& R=\frac{K_{T}^{\chi+2}(\chi+1)[(\chi+1)+\beta(\chi+2)]^{\chi+1}}{F_{S}^{\chi+1}(\beta+1) \chi+2(\chi+2) \chi+1} \\
& \phi_{\max }=\frac{F_{s}(\beta+1)(\chi+2)}{K_{T}[(\chi+1)+\beta(\chi+2)]} \quad S=\frac{\beta}{1+\beta} K_{T}-k_{a}
\end{aligned}
$$


Let the following normalization parameters be $F(u) / F_{s}=p, u / \phi_{\max }=q$. The normalized backbone curve expression is shown in Eq. (3.8), where $q_{\max }$ is the amplitude of normalized excitation. When $q_{\max } \geqslant 1$, the residual stiffness is characterized due to stiffness of the linear spring

$$
p(q)= \begin{cases}\frac{(\chi+2)(\beta+1) q-q^{\chi+2}}{\chi+1+\beta(\chi+2)} & q_{\max }<1 \\ 1+k_{a}(q-1) & q_{\max } \geqslant 1\end{cases}
$$

In order to analysis the influence of $\beta$, we have drawn three representative curves of three different values of $\beta$ with a constant value of the residual stiffness $k_{a}$ as shown in Fig. 6 . As the value of $\beta$ increases, the initial stiffness decreases, but the stiffness of the microslip end increases. $\beta=0$ and $\beta=\infty$ are two extreme cases. In the first case, the initial stiffness is the largest, and the end stiffness is zero. In the second case, the initial stiffness is the smallest, and the stiffness is kept constant throughout the entire microslip. It can be seen that $\beta$ is a parameter controlling the nonlinearity and determining the stiffness continuity.

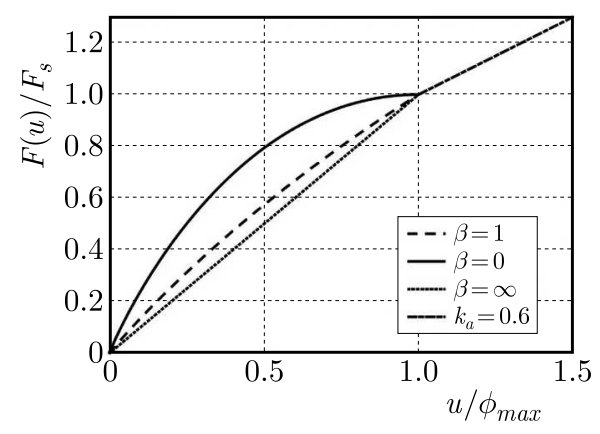

Fig. 6. Normalized backbone curve of the five-parameter Iwan model $\chi=-0.5$

The above research focuses on the backbone curve of the bolted joint. However, bolts are more susceptible to vibration loads. Therefore, it is necessary to study the restoring force during the loading and unloading process under a harmonic load.

During the initial loading process, there are two states for Jenkins elements. Some elements are in the yielding state when $u>\phi$, providing the restoring force as $f^{*} / N$. The rest elements are in the elastic state when $u<\phi$, providing the restoring force as $u k_{r} / N$.

In the unloading process, there are three states. Let $A$ represents the amplitude of displacement $u$. The Jenkins spring element stretches from the loading direction to the unloading direction, and there is an elastic range of $2 \phi$ in length. Some elements are always in the elastic state because $A<\phi$. Some elements are in the elastic state when $A-u<2 \phi$. The rest Jenkins elements yield when $A-u>2 \phi$. There is also a linear spring that is always in the elastic state. Therefore, the restoring force during the unloading stage consists of four parts

$$
\overleftarrow{f}_{m i c}(u)=\int_{0}^{\frac{A-u}{2}}-\phi \rho(\phi) d \phi+\int_{\frac{(A-u)}{2}}^{A}(u-A+\phi) \rho(\phi) d \phi+\int_{A}^{\infty} u \rho(\phi) d \phi+k_{a} u
$$

The restoring force in the loading process is

$$
\vec{F}_{\text {mic }}(u)=\int_{0}^{\frac{A+u}{2}} \phi \rho(\phi) d \phi+\int_{\frac{A+u}{2}}^{A}(u+A-\phi) \rho(\phi) d \phi+\int_{A}^{\infty} u \rho(\phi) d \phi+k_{a} u
$$


Substituting Eq. (3.2) into Eqs. (3.9) and (3.10), we can get

$$
\begin{aligned}
& \overleftarrow{F}_{\text {mic }}(u)=\frac{R(A-u)^{\chi+2}}{2^{\chi+1}(\chi+1)(\chi+2)}-\frac{R A^{\chi+2}}{(\chi+1)(\chi+2)}+\frac{R \phi_{\text {max }}^{\chi+1}}{\chi+1} u+S u+k_{a} u \\
& \vec{F}_{m i c}(u)=-\frac{R(A+u)^{\chi+2}}{2^{\chi+1}(\chi+1)(\chi+2)}+\frac{R A^{\chi+2}}{(\chi+1)(\chi+2)}+\frac{R \phi_{\text {max }}^{\chi+1}}{\chi+1} u+S u+k_{a} u
\end{aligned}
$$

The highest power of the displacement $u$ is $\chi+2$, which means that the hysteretic loop is nonlinear.

The hysteresis curve is normalized the same way as the backbone curve, and the relationship between $p$ and $q$ is

$$
\begin{aligned}
& \overleftarrow{p}_{\text {mic }}(q)=\frac{-m^{\chi+2}+(m-q)^{\chi+2} / 2^{\chi+1}+(q+\beta q)(\chi+2)}{\beta(\chi+2)+\chi+1} \\
& \vec{p}_{\text {mic }}(q)=\frac{m^{\chi+2}-(m+q)^{\chi+2} / 2^{\chi+1}+(q+\beta q)(\chi+2)}{\beta(\chi+2)+\chi+1}
\end{aligned}
$$

Figure 7 shows the normalized hysteresis curve of microslip. It can be found that as $\beta$ decreases, the area enclosed by the hysteresis curve becomes larger.

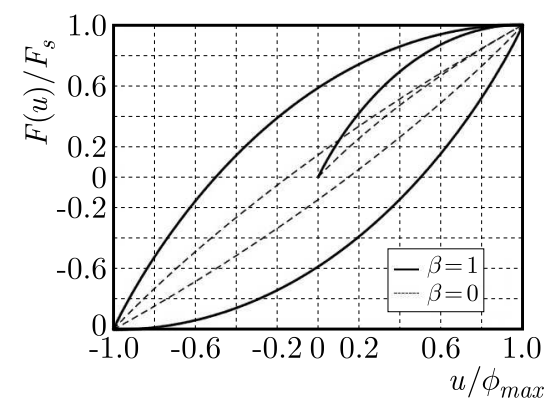

Fig. 7. Normalized hysteresis curve of microslip

When the displacement amplitude of the excitation is greater than the maximum yield displacement of Jenkins elements, the slip shifts from the microslip to the macroslip stage. The restoring force during the unloading process is mainly divided into the nonlinear section and linear section. Only a part of Jenkins elements yield in the nonlinear stage, and the system enters the linear stage when all Jenkins elements yield. The restoring force in the nonlinear stage when $A-2 \phi_{\max } \leqslant u \leqslant A$ is

$$
\begin{aligned}
& \overleftarrow{F}_{\text {mac }}(u)=\int_{0}^{\frac{A-u}{2}}-\phi \rho(\phi) d \phi+\int_{\frac{A-u}{2}}^{\infty}(u-A+\phi) \rho(\phi) d \phi+k_{a} u \\
& =\frac{R(A-u)^{\chi+2}}{2^{\chi+1}(\chi+1)(\chi+2)}-\frac{R \phi_{\max }^{\chi+1}(A-u)}{\chi+1}+\frac{R \phi_{\max }^{\chi+2}}{\chi+2}-S(A-u)+S \phi_{\max }+k_{a} u
\end{aligned}
$$

The restoring force in the linear stage when $-A \leqslant u<A-2 \phi_{\max }$ is

$$
\overleftarrow{F}_{\operatorname{mac}}(u)=\int_{0}^{\phi_{\max }}-\phi \rho(\phi) d \phi-\int_{\phi_{\max }}^{\infty} \phi \rho(\phi) d \phi+k_{a} u=-\frac{R \phi_{\max }^{\chi+2}}{\chi+2}-S \phi_{\max }+k_{a} u
$$


The restoring force during the loading process of the hysteresis curve can be derived from the Masing criterion

$$
\vec{F}_{\operatorname{mac}}(u)= \begin{cases}-\frac{R(A+u)^{\chi+2}}{2^{\chi+1}(\chi+1)(\chi+2)}+\frac{R \phi_{\max }^{\chi+1}(A+u)}{\chi+1}-\frac{R \phi_{\max }^{\chi+2}}{\chi+2} & \\ \quad+S(A+u)-S \phi_{\max }+k_{a} u & -A \leqslant u \leqslant 2 \phi_{\max }-A \\ \frac{R \phi_{\max }^{\chi+2}}{\chi+2}+S \phi_{\max }+k_{a} u & 2 \phi_{\max }-A<u \leqslant A\end{cases}
$$

We select a set of parameters $\chi=-0.5, \beta=0.1, k_{a}=1, R=5.5, \phi_{\max }=1, A=1.5$ to plot the hysteresis curve as shown in Fig. 8. By comparing with Gaul's experimental results, we find that the curve generated by the five-parameter model is similar to Gaul's experimental curve.

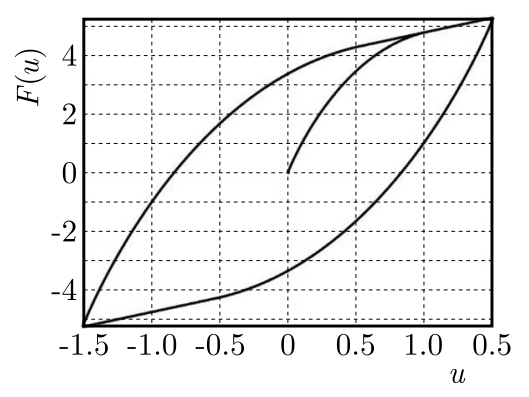

Fig. 8. Hysteresis curve of macroslip

In a single excitation cycle, the energy dissipation is equal to the area enclosed by the loading curve and the unloading curve, which can be calculated through

$$
D(A)=\int_{-A}^{A}[\vec{F}(u)-\overleftarrow{F}(u)] d u
$$

Substituting Eqs. (3.11) and (3.13)-(3.15) into Eq. (3.16), we can get a relationship between the energy dissipation and displacement amplitude

$$
\begin{aligned}
& D_{\operatorname{mic}}(A)=\frac{4 R A^{\chi+3}}{(\chi+2)(\chi+3)}=w A^{\chi+3} \quad A<\phi_{\max } \\
& D_{\operatorname{mac}}(A)=\frac{4 R A \phi_{\max }^{x+2}}{x+2}+4 S A \phi_{\max }-\frac{4 R \phi_{\max }^{\chi+3}}{\chi+3}-4 S \phi_{\max }^{2} \quad A \geqslant \phi_{\max }
\end{aligned}
$$

The backbone curve and the hysteresis curve of microslip are normalized above. In order to unify and simplify the macroslip curve and the energy dissipation relationship, we propose a new set of dimensionless variables to simplify the equations. $\stackrel{\vec{P}}{=} \stackrel{\vec{F}}{F}(u) /\left(k_{a} \phi_{\max }\right)$ is the dimensionless restoring force. $Q=u / \phi_{\max }$ is the dimensionless displacement. $\kappa_{1}=R \phi_{\max }^{\chi+1} / k_{a}$ and $\kappa_{2}=S / k_{a}$ are the inherent dimensionless stiffness parameters of the system. $\eta=A / \phi_{\max }$ is the dimensionless amplitude. $P_{\text {init }}$ represents the dimensionless restoring force in the initial loading process

$$
P_{\text {init }}(Q)= \begin{cases}\left(\frac{\kappa_{1}}{\chi+1}+\kappa_{2}+1\right) Q-\frac{\kappa_{1}}{Q^{\chi+2}}(\chi+1)(\chi+2) & Q<1 \\ Q+\kappa_{2}+\frac{\kappa_{1}}{\chi+2} & Q \geqslant 1\end{cases}
$$


The restoring force in the hysteresis curve of microslip and macroslip, expressed in the dimensionless form for $-\eta \leqslant Q \leqslant \eta$

$$
\begin{aligned}
& \overleftarrow{P}_{\text {mic }}(Q)=\frac{\kappa_{1}}{(\eta-Q)^{\chi+2}} 2^{\chi+1}(\chi+1)(\chi+2)+\left(\frac{\kappa_{1}}{\chi+1}+\kappa_{2}+1\right) Q-\frac{\kappa_{1} \eta^{\chi+2}}{(\chi+1)(\chi+2)} \\
& \vec{P}_{\text {mic }}(Q)=-\frac{\kappa_{1}}{(\eta+Q)^{\chi+2}} 2^{\chi+1}(\chi+1)(\chi+2)+\left(\frac{\kappa_{1}}{\chi+1}+\kappa_{2}+1\right) Q+\frac{\kappa_{1} \eta^{\chi+2}}{(\chi+1)(\chi+2)}
\end{aligned}
$$

and

$$
\begin{aligned}
& \overleftarrow{P}_{m a c}(Q)=\left\{\begin{array}{cc}
\frac{\kappa_{1}(\eta-Q)^{\chi+2}}{2^{\chi+1}(\chi+1)(\chi+2)}-\left(\frac{\kappa_{1}}{\chi+1}+\kappa_{2}\right)(\eta-Q) & \\
+Q+\frac{\kappa_{1}}{\chi+2}+\kappa_{2} & \eta-2 \leqslant Q \leqslant \eta \\
Q-\frac{\kappa_{1}}{\chi+2}-\kappa_{2} & -\eta<Q<\eta-2
\end{array}\right. \\
& \vec{P}_{\text {mac }}(Q)= \begin{cases}-\frac{\kappa_{1}(\eta+Q)^{\chi+2}}{2 \chi+1}\left(\chi+\frac{1}{\kappa_{1}(\chi+2)}+\left(\frac{\kappa_{1}}{\chi+1}+\kappa_{2}\right)(\eta+Q)\right. & \\
+Q-\frac{\kappa_{1}}{\chi+2}-\kappa_{2} & -\eta \leqslant Q \leqslant 2-\eta \\
Q+\frac{\kappa_{1}}{\chi+2}+\kappa_{2} & 2-\eta<Q \leqslant \eta\end{cases}
\end{aligned}
$$

Let $d_{\text {mic }}(\eta)=D_{\text {mic }}(A) /\left(k_{a} \phi_{\max }^{2}\right)$ express the dimensionless energy dissipation. We can get the dimensionless relationship between the energy dissipation and displacement in micro and macro slip

$$
\begin{aligned}
& d_{\text {mic }}(\eta)=\frac{4 \kappa_{1} \eta^{\chi+3}}{(\chi+2)(\chi+3)} \quad \eta<1 \\
& d_{\text {mac }}(\eta)=\left(\frac{4 \kappa_{1}}{\chi+2}+4 \kappa_{2}\right) \eta-\left(\frac{4 \kappa_{1}}{\chi+3}+4 \kappa_{2}\right) \quad \eta \geqslant 1
\end{aligned}
$$

From Eq. (3.21) 1 , we can see that the dimensionless energy dissipation of microslip has a log$\log$ linear relationship with the excitation amplitude. The slope is $\chi+3$ in the double logarithmic coordinate system, which is consistent with the results in the literature.

Figure 9 shows the curve of dimensionless dissipation of different $\kappa_{1}$ in the linear coordinate and double logarithmic coordinate system, respectively.

(a)

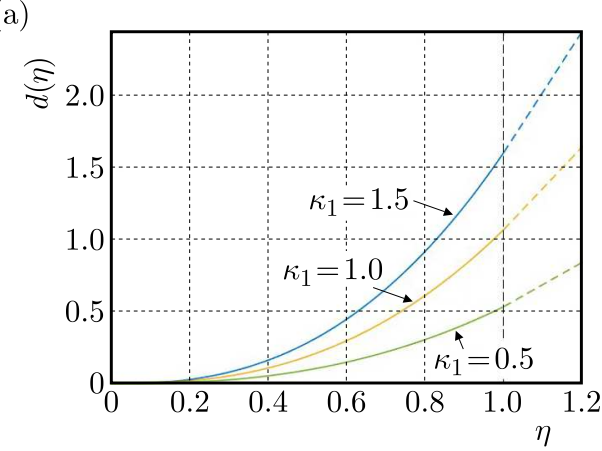

(b)

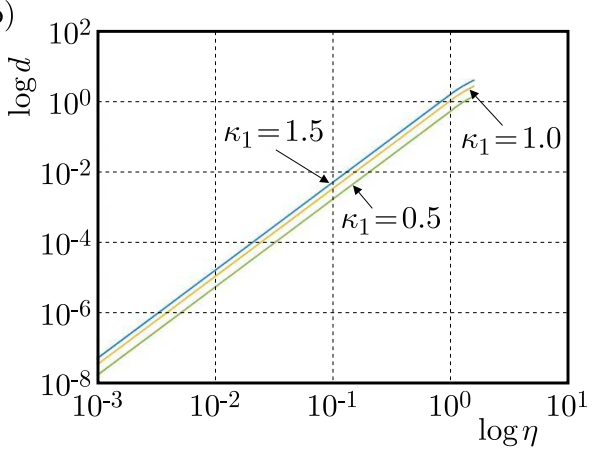

Fig. 9. Dimensionless dissipation curve $\left(\chi=-0.5, \kappa_{2}=0.05\right)$ : (a) linear coordinate, (b) double logarithmic coordinate system

It can be found from Fig. 9 that although there is no stuck state in the strict sense according to the theory of this model, in the initial stage of the dimensionless displacement the energy dissipation value is very close to zero but not zero, which can be approximated by a linear 
relationship between the restoring force and displacement. This phenomenon is consistent with the literature (Segalman et al., 2009) that the response appears linear at very low loads, and even at this point there is some microslip and dissipation. In the double logarithmic coordinates, the non-dimensional energy dissipation of microslip has a linear relationship with the dimensionless amplitude. On the premise of keeping $\kappa_{2}$ unchanged, the energy dissipation will increase with an increase of $\kappa_{1}$.

Figure 10 shows the relationship between the parameter $\chi$ and the energy dissipation. The increase of $\chi$ will reduce the energy dissipation. And, as $\eta$ increases, the curves for different $\chi$ values approach each other in the double logarithmic coordinates.

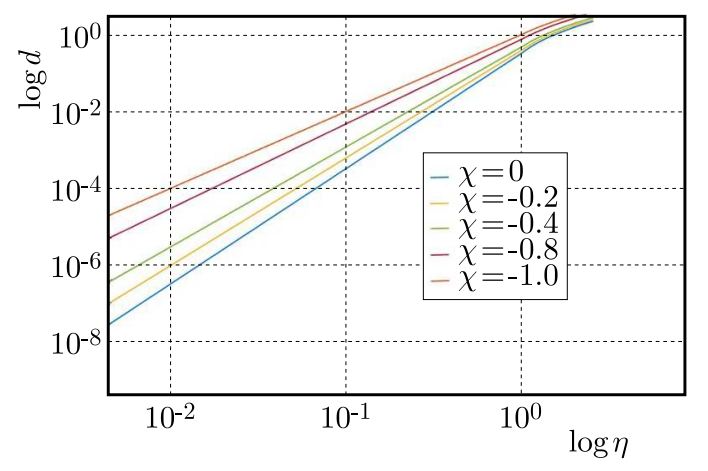

Fig. 10. Energy dissipation curve for different $\chi$

\section{Modified four-parameter model with further consideration of stiffness continuity}

The five-parameter model proposed above still exhibits stiffness discontinuity at the macroslip point, which is contrary to experimental results of different excitation forces (Gaul and Lenz, 1997) and simulation results of different pre-tightening forces and friction coefficients (Zhao et al., 2021) as shown in Fig. 11. It shows that the stiffness degradation should be continuous.

Through the analysis of Eq. (3.4) and Fig. 6, we find that the discontinuity is caused by the value of $\beta$. When $S$ is equal to zero, there will be no stiffness discontinuity at the macroslip point. Therefore, the modified four-parameter model is further studied. Compared with the five-parameter model, the justified four-parameter model is a special case of $S=0$, and the corresponding restoring force calculation formula will not be presented here. The focus is the characteristic of energy dissipation. In the nondimensionalization, $S=0$ is equivalent to $\kappa_{2}=0$

$$
\begin{array}{ll}
d_{\text {mic }}(\eta)=\frac{4 \kappa_{1} \eta^{\chi+3}}{(\chi+2)(\chi+3)} & \eta<1 \\
d_{m a c}(\eta)=\frac{4 \kappa_{1}}{\chi+2} \eta-\frac{4 \kappa_{1}}{\chi+3} & \eta \geqslant 1
\end{array}
$$

It can be seen from Eq. (4.1) 1 that the dimensionless energy dissipation still maintains a linear relationship with a slope of $\chi+3$ with the dimensionless displacement amplitude in the double logarithmic coordinate system.

The interface wear caused by different excitation frequencies in a short time is different, but the impact of wear can be ignored and is usually not considered in the current bolt dynamics research. The resonance frequency of the bolt assembly is always selected as the loading frequency for more obvious energy dissipation under the same load amplitude. A set of experimental data for stepped specimens performed by Segalman with the Big Mass Device is selected as a comparison. 


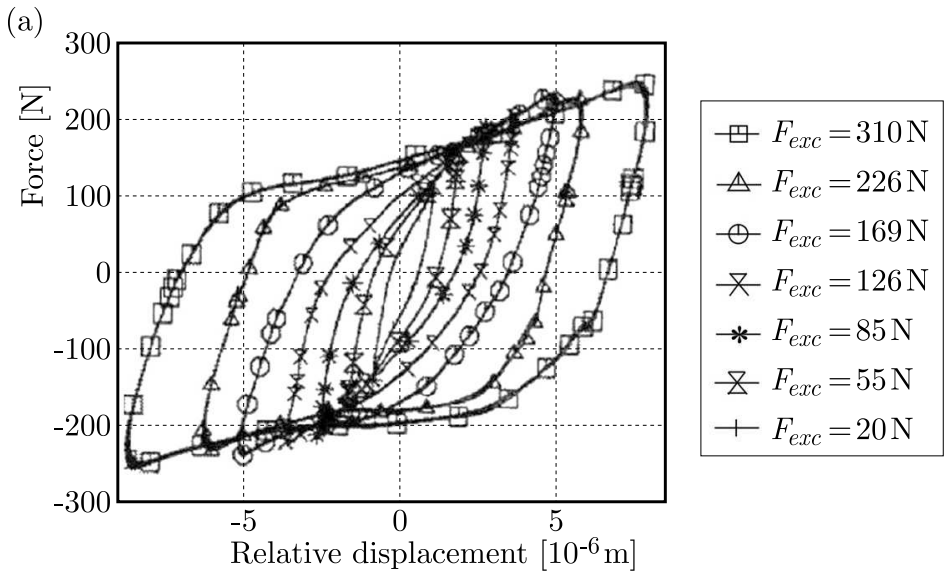

(b)

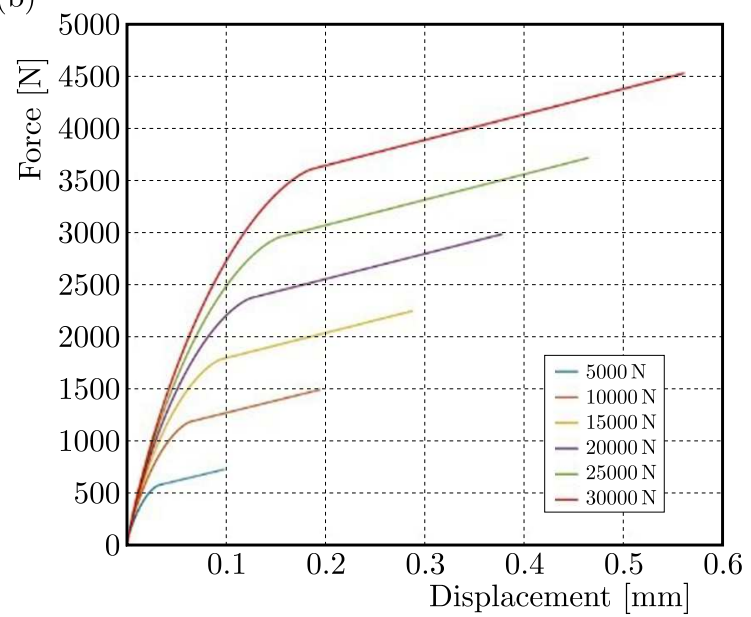

(c)

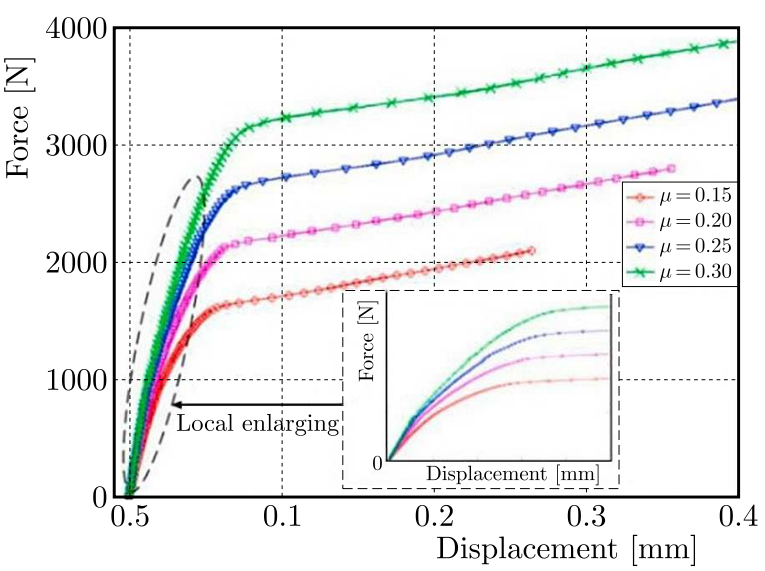

Fig. 11. Experimental and simulation results of the force-displacement curve: (a) experimental curves, (b) simulation curves, (c) simulation curves of different friction coefficients

Figure 12 shows that the curve fitted by the least square method is in good agreement with the data. The slope in the double logarithmic coordinate system of the fitted curve is 2.8916, which is also in good agreement with the slope of 2.8272 in Segalman's experiments.

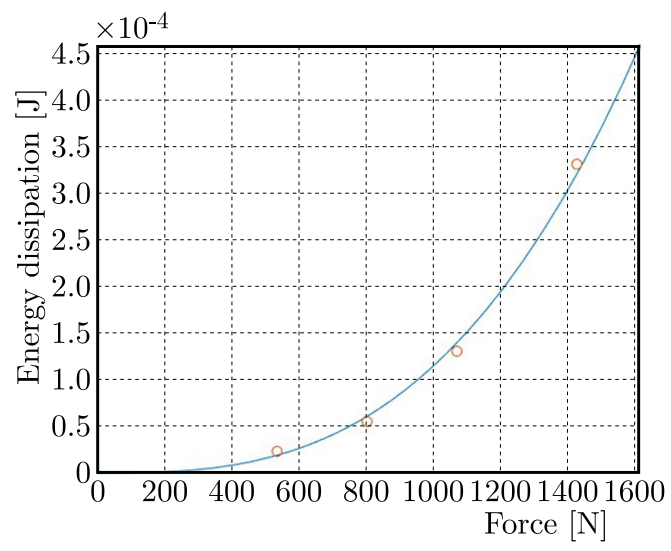

Fig. 12. Energy dissipation curve fitted with the modified four-parameter model: $R=97.5837$,

$$
\phi_{\max }=-0.009, \chi=-0.1084, k_{a}=100790
$$




\section{Conclusion}

- In this article, several typical Iwan models are analyzed, and a five-parameter model combining Jenkins elements, a linear spring and the truncated power-law distribution is proposed, which is able to characterize residual stiffness.

- The restoring force-displacement functions of the five-parameter model backbone curve and the microslip hysteresis curve are normalized, and the influence of $\beta$ is observed. The value of $\beta$ is the determining factor for the system stiffness discontinuity.

- The restoring force calculation equations of the five-parameter model are dimensionlessly processed, and the influence of $\chi$ and $\kappa_{1}$ on energy dissipation is analyzed. A decrease in the value of $\chi$ or an increase in the value of $\kappa_{1}$ will lead to the increase of energy dissipation.

- On the basis of the five-parameter model, a modified four-parameter model with the stiffness continuity is proposed, and the dimensionless energy dissipation relationship is derived using the same dimensionless processing method. And its effectiveness has been verified by experimental data.

- The justified four-parameter model proposed in this paper adds constraints to the variable $S$ to characterize the stiffness continuity, and at the same time the number of model parameters is reduced and the model is simplified. This model is considered to be beneficial for engineering applications such as discrete analysis and finite element calculations.

\section{Acknowledgements}

The equipment pre-research fund (41426030107) supporting the completion of this project is acknowledged.

\section{References}

1. Argatov I.I., Butcher E.A., 2011, On the Iwan models for lap-type bolted joints, International Journal of Non-Linear Mechanics, 46, 2, 347-356

2. Beards C.F., 1992, Damping in structure joints, Shock and Vibration Digest, 24, 3-7

3. BickFord J.H, 2007, Introduction to the Design and Behavior of Bolted Joints: Non-Gasketed Joints, CRC Press

4. CaO J., Liu Q., Hong J., 2020, A review of the micro-to-macro dynamics of bolted connection, China Mechanical Engineering, 1-14

5. Ferri A.A., 1995, Friction damping and isolation systems, Journal of Mechanical Design, 117, B, 196-206

6. Gaul L., Lenz J., 1997, Nonlinear dynamics of structures assembled by bolted joints, Acta Mechanica, 125, 1, 169-181

7. Goodman L.E., 1959, A Review of Progress in Analysis of Interfacial Slip Damping, https://books.google.pl/books?id=jaWLygAACAAJ

8. IwAN W.D., 1966, A distributed-element model for hysteresis and its steady-state dynamic response, Journal of Applied Mechanics, 33, 4

9. LI D., Xu C., 2017, Iwan model considering normal load changes and its characteristics analysis, Journal of Harbin Institute of Technology, 49, 10, 138-144

10. Li Y., HaO Z., Zhang D., 2015, Research on Iwan model based on six-parameter non-uniform density function, Chinese Journal of Theoretical and Applied Mechanics, 47, 3, 513-520

11. Liu B., Dong X., Peng Z., 2020, Nonlinear equivalent model of bolted joint joint surface based on Iwan model, Noise and Vibration Control, 40, 2, 7-12 
12. Mindlin R.D., 1949, Compliance of elastic bodies in contact, Journal of Applied Mechanics ASME, 16, 259-268

13. Mindlin R.D., 1952, Effects of an oscillating tangential force on the contact surfaces of elastic spheres, Proceedings of 1st US National Congress of Applied Mechanics, ASME, New York, 203

14. Segalman D.J., Gregory D.L., Starr M.J., Resor B.R., Jew M.D., Lauffer J.P., Ames N.M., 2009, Handbook on Dynamics of Joints Structures, Technical Report SAND2009-4164, Sandia National Laboratories, NM, USA

15. Song Y., Hartwigsen C.J., McFarland D.M., Vakakis A.F., Bergman L.A., 2004, Simulation of dynamics of beam structures with bolted joints using adjusted Iwan beam elements, Journal of Sound and Vibration, 273, 1, 249-276

16. WANG D., FAN X., 2019, Dynamic analysis of hysteresis and nonlinear characteristics of connection interface, Journal of Vibration Engineering, 32, 6, 1060-1066

17. Zhang X., Wang B., Wei H., 2012, Non-linear restoring force and energy dissipation calculation of Iwan model, Engineering Mechanics, 29, 11, 33-39

18. Zhao B., Wu F., Sun K., Mu X., Zhang Y., Sun Q., 2021, Study on tangential stiffness nonlinear softening of bolted joint in friction-sliding process, Tribology International, 156, 106856

Manuscript received June 15, 2021; accepted for print October 4, 2021 